\title{
Exploring Edge-Based Input Techniques for Handheld Text Entry
}

\author{
Jacob O. Wobbrock, Brad A. Myers, and Scott E. Hudson \\ Human Computer Interaction Institute \\ School of Computer Science \\ Carnegie Mellon University \\ Pittsburgh, PA 15213 \\ \{ jrock, bam, scott.hudson \}@cs.cmu.edu
}

\begin{abstract}
We are investigating how handheld devices like Palm PDAs and PocketPCs can be used as assistive technologies for computer access by people with motor impairments such as Muscular Dystrophy and Cerebral Palsy. As part of this research, we are developing new input techniques for handheld text entry. People with motor impairments suffer from symptoms that affect their ability to use conventional text entry methods. One symptom is a lack of stability in stylus movements caused by tremor or spasm. In an effort to create a more stable means of text entry, we are researching how to leverage elevated physical edges in our development of new text entry techniques. We present three edge-based techniques: Edge Keyboards, CornerSlide, and EdgeWrite.
\end{abstract}

\section{Introduction}

People with motor impairments often lose their capacity for gross motor movement before they lose fine motor control [11]. This often inhibits their use of a keyboard and mouse. Even those with motor impairments who can use a keyboard and mouse often fatigue quickly due to their limited muscle strength and dexterity.

The field of computer access is concerned with providing technologies that make computer use possible and comfortable for people with special needs. However, specialized computer access devices are often expensive and require configuration by a specialist [2]. Rather than develop new devices for computer access, we have focused on leveraging existing consumer-grade handheld devices for computer access. These devices-Palm PDAs and PocketPCs - have the advantage in that they are small, so people with motor impairments can often move a stylus or fingertip across the screen. Furthermore, they are programmable, so we can create software that is adaptive to the needs of the user and their particular abilities. Handheld devices are also relatively inexpensive and require little setup or maintenance.

An application we have developed as part of the Pebbles project (www.cs.cmu.edu/ pebbles/) called Remote Commander enables people to control their desktop computers entirely from a connected handheld device [12]. Some people with Muscular Dystrophy have already found this application useful for computer access, in part because it extends their longevity of use due to decreased fatigue [18]. For the details of this software and user study results, the reader is directed to a prior article [13].

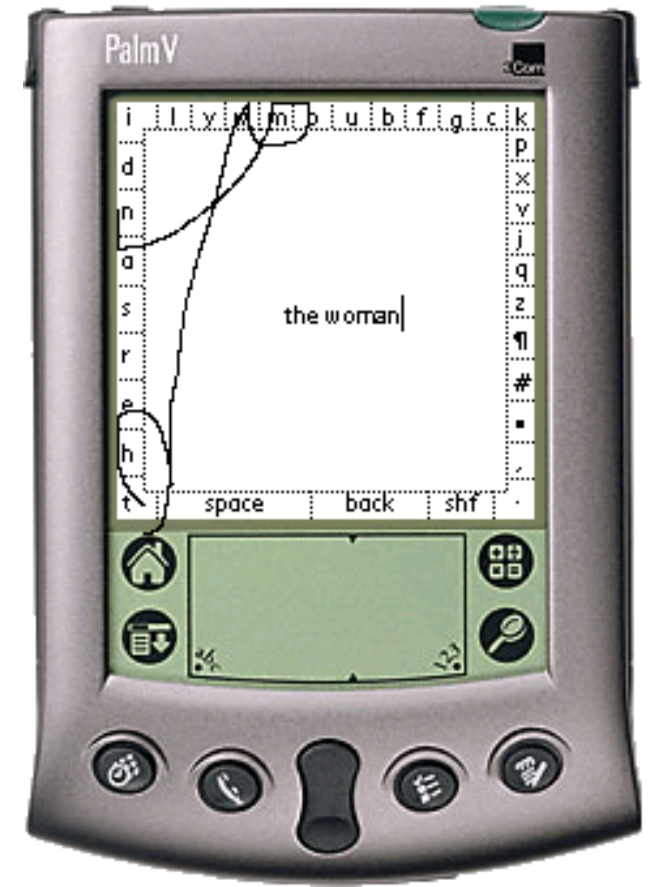

Figure 1. One of our four Edge Keyboard designs. These continuous-gesture keyboards send input when the edge behind a letter-region is impacted by a stylus, or when a letter region is tapped. The stroke above writes "the woman".

Despite the early success of this research, problems still exist, particularly in the area of text entry. Text entry on handheld devices is an active area of research [9], but none of it has focused on text entry by people with motor impairments. Current handheld input techniques such as the various unistroke alphabets (e.g., Graffiti and Jot) and on-screen keyboards can be difficult for people with motor impairments, who may lack stability in their motions due to intention tremor, essential tremor, or spasms [14].

Our current research goal is to develop new text entry techniques that increase stability by leveraging elevated physical edges, such as those found around the edges of a handheld screen, or those added by a small plastic attachment. Edges also have a promising Fitts' Law benefit for making targets easier to acquire [3][8]. Here we present three of our working ideas for this research: Edge Keyboards, CornerSlide, and EdgeWrite. Ultimately, we want to develop better models of human motion and of the abilities of motor-impaired users. Such models would aid design efforts in helping to predict human performance. 


\section{Related Work}

The related work falls largely into two areas: handheld text entry and computer access. While there is a good deal of work in both areas, there is little that sits at the seam between the two. An exception is the Archimedes Project [15], though the handheld they are using is a custom device, not a consumergrade PDA like we are using.

Text entry on handhelds has been studied extensively (e.g., [17]), including the development of optimal keyboard layouts [20]. Two non-keyboard input techniques are Quikwriting [16] and Cirrin [10], which use continuous gestures through zones. One of the few examples of explicit edge-based research placed Web browser controls at the edges of a desktop computer screen and measured the Fitts' Law benefits [1].

A good deal of research exists in computer access and modeling user motion, some modeling the motion of motorimpaired users. Examples are techniques for evaluating problems with keyboard configurations [19], keystroke-level models of word prediction for people with disabilities [7], and the work of the Usability and Rehabilitation Engineering Group at Cambridge University, which has shown that models of user behavior for motor-impaired users need to be different than those for able-bodied users [5][6].

\section{Edge Keyboards}

Pilot studies we conducted with Remote Commander showed that some users with Muscular Dystrophy could use a stylus with a handheld device to tap keys on a virtual keyboard [13]. However, text entry was slow and target acquisition difficult for many subjects. We observed that many of the subjects held the stylus at a steep angle so that the tip of the stylus was easily pushed against the elevated left edge, where the screen met the physical encasing for the device.

The idea for Edge Keyboards grew from these observations and the notion that targets against an edge are easier to acquire because the edge allows for effective target overshooting [1]. We built four versions of continuous-gesture edge keyboards: 1- and 2-screen version of both frequency-based and alphabetic layouts. We tried them in a pilot study with 10 ablebodied users, and our results showed that after five minutes of practice, users could enter about $10.2 \mathrm{wpm}$ with the design in Figure 1, compared to about $12.4 \mathrm{wpm}$ for the same tasks with Graffiti, and $22.2 \mathrm{wpm}$ for on-screen QWERTY. Performing more formal evaluations and evaluations with motor-impaired users are part of our ongoing work.

\subsection{Interaction with Edge Keyboards}

A character is entered on an Edge Keyboard when the stylus impacts the elevated physical edge of the handheld device behind the letter region (Figure 1). Along the bottom row, where there is no edge, users can simply overshoot the space, backspace, and shift regions. All regions can also be tapped like conventional soft buttons. Users can enter text with the edge keyboards without ever lifting their stylus. This may provide benefits to users with motor impairments, as they can rest the stylus on the surface of the PDA during text entry.

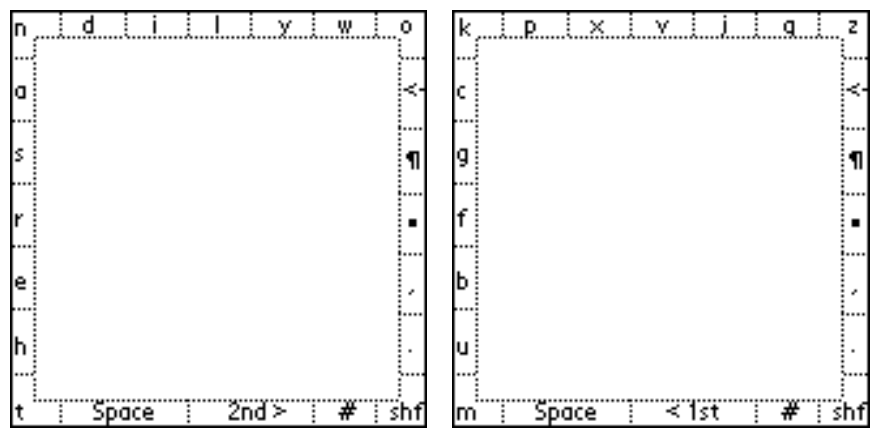

Figure 2. In this 2-Screen design, each screen contains half the letters in the alphabet, and users switch between them by tapping or swiping over the "2nd >" and "< 1st" regions.

Other user interface elements could be placed in the center of the screen if desired, since once a user begins a gesture across the keyboard, only keyboard input will be sent until the user lifts the stylus. Numbers and punctuation can be accessed in this design via the "\#" region and the "." region, which transition to other screens with similarly laid-out numbers and punctuation marks.

\subsection{The Other Three Edge Keyboard Designs}

The design shown in Figure 1 is a Thersandily layout, named for the order of letters starting from the lower-left. The layout of letters in this design was determined by the use of a simulated annealing algorithm that optimized the distance between common letter-pairs from a large corpus of text [17]. One can see that common words like "the" and "and" can be made by simply sliding the stylus along the edge across the appropriate letter regions.

A difficulty with the Thersandily layout is the small letter regions. For this reason, we developed a 2 -screen version of the Thersandily layout, and transitions were made between screens by "swiping over" a region in the bottom row (Figure 2 ). The 2-screen version devotes more edge space to each letter, but at a cost of having to transition to the less-frequently used letters on the second screen. In our pilot studies, users entered text at about $8.3 \mathrm{wpm}$ with the 2 -screen version.

The other two designs we explored were 1- and 2-screen alphabetic layouts. Despite the intuition that users might perform better given an alphabetic layout due to familiarity, our pilot studies showed that users do not benefit from alphabetic layouts, as the exact order of letters is not readily obvious. Given enough practice to become familiar with the layout, frequency-based designs seem the clear choice over alphabetic designs. In general, the main challenge of using these designs seemed to be the cognitive load endured by users as they searched for the next letter. This presumably would decrease with more practice and familiarity. In the one hour span of our pilot study, all of the 10 users became more fluid in their gestures and quicker to find the next letter.

\section{Other Edge-Based Techniques}

We are actively exploring two other edge-based input techniques tailored to those with motor impairments. 


\subsection{Interaction at the Corners: CornerSlide}

If edges are easy targets, then corners are even easier. Corners benefit from edges in two directions, and naturally "pocket" a stylus that is slid along either edge leading to them. For this reason, we are exploring a corner-oriented input technique called CornerSlide.

A small number of letters can be assigned to the two edges at each of the four corners of a handheld screen, similar to how letters are assigned to telephone buttons. (Of eight cornerforming edges, two get four letters, six get three letters.) A user slides into a corner along an edge one, two, or three times to access the first, second, and third letters, respectively. When the user moves away from the edge or to another corner, the current letter is committed. Spaces and punctuation reside halfway along each edge between corners for easy access "on the way" from one corner to another. This technique may help those users with motor impairments who can achieve no better accuracy than to put the stylus somewhere on the handheld screen and slide it along an edge into a corner.

\subsection{Edge-Guided Characters: EdgeWrite}

We are currently investigating how an alphabet whose letter-forms are defined along the interior edges of a small plastic square hole might be of help to users who have difficulty making smooth gestures, such as the gestures required by Graffiti. A plastic attachment sits over the Graffiti area of a Palm and contains square hole to guide the stylus in straight lines. Other research has shown that the four cardinal directions are the easiest gestures to make [4]. The characters in the EdgeWrite alphabet are defined only by the order in which the corners of the square are hit (Figure 3). That is, recognition does not depend on the whole stroke path. This allows users with tremor to deviate from the edge-guide and still complete the letter forms. Most EdgeWrite forms resemble their respective "real letters" in feel. We have implemented EdgeWrite and are in the process of formally evaluating it with able-bodied and motor-impaired users.

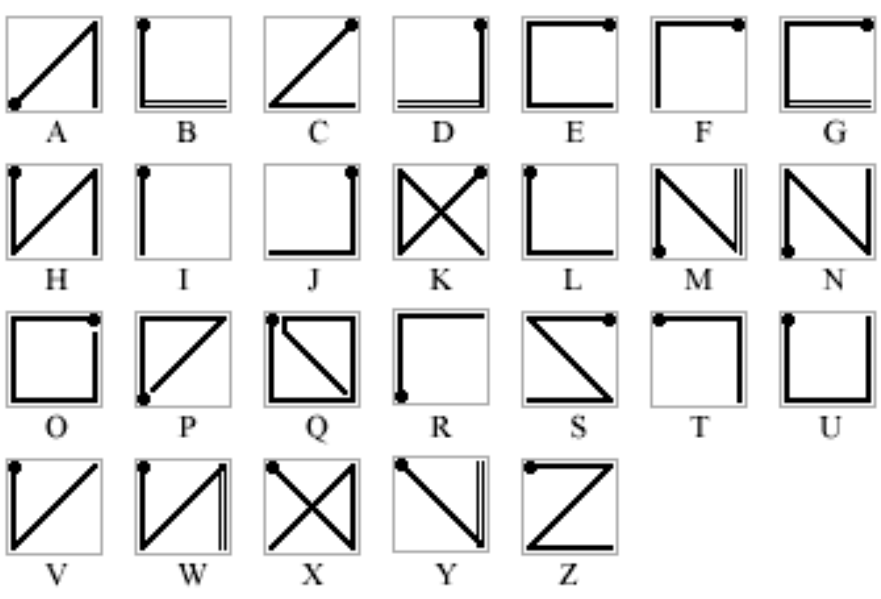

Figure 3. Prototype EdgeWrite alphabet. Double-lines indicate a trace over the same edge. Users are free to depart from the edges as they make strokes because only the order in which the corners are hit defines the letter forms. Many letters have alternates not shown here.

\section{Conclusions}

We believe that physical edges hold promise for text entry for people with motor impairments because of increased stability and easier target acquisition. In addition, these techniques may benefit all users of mobile technology with improved input techniques. Ultimately, we want to model motor-impaired stylus behavior in order to give the exploration of techniques such as these a more empirical foundation from which to build.

\section{References}

[1] Farris, J.S., Jones, K.S., Anders, B.A. (2001) Acquisition speed with targets on the edge of the screen: An application of Fitts' Law to commonly used web browser controls. Proc. Human Factors and Ergonomics Society 45th Annual Meeting, 1205-1209.

[2] Fichten, C.S., Barile, M., Asuncion, J.V., Fossey, M. (1999) What government and organizations which help postsecondary students obtain computer, information and adaptive technologies can do to improve learning and teaching. EvNet Working Papers \#3. Montreal, Quebec.

[3] Fitts, P.M. (1954) The information capacity of the human motor system in controlling the amplitude of movement. Journal of Experimental Psychology 47, 381-389.

[4] Isokoski, P. (1999) A minimal device-independent text input method. Unpublished thesis, University of Tampere, Finland. See Reference 9.

[5] Keates, S., Clarkson, P.J., Robinson, P. (2000) Investigating the applicability of user models for motion-impaired users. Proc. ASSETS 'O0, 129-136.

[6] Keates, S., Clarkson, P.J., Robinson, P. (1998) Developing a methodology for the design of accessible interfaces. Proc. 4th ERCIM Workshop on User Interfaces for All, 1-15.

[7] Koester, H., Levine, S.P. (1994) Validation of a keystroke-level model for a text entry system used by people with disabilities. Proc. ASSETS '94, 115-122.

[8] MacKenzie, I.S. (1992) Fitts' Law as a research and design tool in human-computer interaction. Human-Computer Interaction 7, 91139.

[9] MacKenzie, I.S., Soukoreff, R.W. (2002) Text entry for mobile computing: Models and methods, theory and practice. HumanComputer Interaction 17, 147-198.

[10] Mankoff, J., Abowd, G.D. (1998) Cirrin: A word-level unistroke keyboard for pen input. Proc. UIST '98, 213-214.

[11] MDA. The Muscular Dystrophy Association. (2001) Available at http://www.mdausa.org/

[12] Myers, B.A. (2001) Using hand-held devices and PCs together. Communications of the ACM 44 (11), 34-41.

[13] Myers, B.A., Wobbrock, J.O., Yang, S., Yeung, B., Nichols, J., Miller, R. (2002) Using handhelds to help people with motor impairments. Proc. ASSETS '02, 89-96.

[14] Neurology Channel. (2002) Movement disorders. Available at http://www.neurologychannel.com/movementdisorders/

[15] Newman, Keith. (2002) The open interface: Beyond keyboards and mice. e.nz Magazine, 6-11.

[16] Perlin, K. (1998) Quikwriting: Continuous stylus-based text entry. Proc. UIST '98, 215-216.

[17] Soukoreff, R.W., MacKenzie, I.S. (1995) Theoretical upper and lower bounds on typing speed using a stylus and soft keyboard. Behavior and Information Technology 14, 370-379.

[18] Stack, J. (2001) Palm Pilot connects girl with classroom. QUEST Magazine 8 (1), 48-49.

[19] Trewin, S., Pain, H. (1998) A model of keyboard configuration requirements. Proc. ASSETS '98, 173-181.

[20] Zhai, S., Hunter, M., Smith, B.A. (2002) Performance optimization of virtual keyboards. Human-Computer Interaction 17, 229-269. 